Service social

The One-Parent Family in the 1980s. Perspectives and Annotated Bibliography 1978-1984, par Benjamin Schlesinger, Toronto, University of Toronto Press, 1985, 284 pages.

\title{
Claude Martin
}

Volume 36, numéro 2-3, 1987

Approches intégrées

URI : https://id.erudit.org/iderudit/706379ar

DOI : https://doi.org/10.7202/706379ar

Aller au sommaire du numéro

Éditeur(s)

École de service social de l'Université Laval

ISSN

1708-1734 (numérique)

Découvrir la revue

Citer ce compte rendu

Martin, C. (1987). Compte rendu de [The One-Parent Family in the 1980s.

Perspectives and Annotated Bibliography 1978-1984, par Benjamin Schlesinger,

Toronto, University of Toronto Press, 1985, 284 pages.] Service social, 36(2-3),

501-504. https://doi.org/10.7202/706379ar d'utilisation que vous pouvez consulter en ligne.

https://apropos.erudit.org/fr/usagers/politique-dutilisation/ 
En mineure, et les éléments précédents y demeurant présents, ce rapport se révèle parfois l'occasion d'une rencontre réelle entre deux personnes qui sont aussi en quête d'une présence humaine".

Ici, la prostitution prend un sens tactique à la fois sexuel, économique et social qui décape la conscience individuelle et sociale et nous projette en dehors des clichés habituels, au-delà de l'interprétation réduite de la " passe pour l'argent ". Prostituée - client - souteneur ne sont plus les seuls en cause, mais un large milieu socio-économique et culturel soutient, organise, promeut et contrôle leur relation.

L'œuvre de Nadeau permet l'ouverture d'une parenthèse plus élargie sur un segment de la condition humaine agissante.

École de service social,

Germain TrottieR

Université Laval.

The One-Parent Family in the 1980s. Perspectives and Annotated Bibliography 1978-1984, par Benjamin SCHLESINGER, Toronto, University of Toronto Press, 1985, 284 pages.

L'ouvrage de Schlesinger est d'un genre littéraire relativement rare en Amérique du Nord et quasi-inexistant en Europe. Il n'en est que plus précieux, et ce tout particulièrement pour les chercheurs ou étudiants européens qui méconnaissent le plus souvent l'énorme somme de travaux produits par leurs collègues d'outre-Atlantique. En effet, il s'agit d'un inventaire aussi large et exhaustif que possible des publications (ouvrages, travaux de recherche et articles) concernant les familles monoparentales au Canada et aux États-Unis. On doit avant tout saluer cette initiative de l'auteur, qui depuis la fin des années soixante tient à jour et réédite régulièrement cette bibliographie annotée. On ne peut espérer un outil plus idéal pour traverser cette énorme production nord-américaine. Ainsi conçu, ce document est un outil de travail tout à fait irremplaçable pour toute personne, étudiant, chercheur ou enseignant, intéressé par les questions concernant l'évolution des structures familiales.

La première édition de cet ouvrage date de 1969. Trois autres l'ont suivie et comptaient 750 références bibliographiques annotées portant sur le thème et couvrant la période 1930-1978. La présente édition complète les précédentes en proposant, d'une part, une bibliographie commentée de 490 nouvelles références publiées entre janvier 1978 et juin 1984, et d'autre part, des synthèses de littérature concernant des aspects particuliers liés à la monoparentalité. Ces synthèses rédigées par L. Davids, A.M. Ambert, S.M.H. Hanson, G. Nelson et l'auteur lui-même enrichissent considérablement ce document et montrent l'étendue des questions associées à ce phénomène. Les auteurs ont su dépasser la catégorie elle-même pour aborder des sujets voisins, comme la recomposition des familles, le remariage, ou encore le sujet trop souvent négligé des parents 
gardiens masculins. En plus de l'originalité des thèmes sur lesquels sont effectuées les synthèses, celles-ci ont l'avantage de poser un regard critique sur la littérature recensée, permettant ainsi au lecteur de construire son point de vue, de repérer les écarts, voire les contradictions entre les travaux, mais surtout de préciser les difficultés méthodologiques posées par l'approche des "familles monoparentales".

Il est pratiquement impossible de tenter quelque résumé que ce soit sur une telle somme d'informations. Aussi, nous nous contenterons de donner ici au lecteur quelques indications sur les bilans de littérature.

Dans un premier article, L. Davids fait le point sur l'évolution quantitative du phénomène au Canada, en s'appuyant principalement sur les données fournies par les recensements de population (1971, 1976 et 1981). Y sont adjointes quelques données provenant des Annual Vital Statistics, concernant les taux de naissance, de mariage et de divorce. L'auteur pointe avant tout le fait que, contrairement au passé, l'entrée en situation monoparentale est due de plus en plus souvent aujourd'hui à la dissociation familiale (séparation et divorce); phénomène tout à fait comparable en France. En effet, les taux de divorce ont pratiquement doublé entre 1971 et 1981. Parallèlement, les taux de naissance ont décru tout au long des années soixante-dix, ce qui peut tendre à moduler l'augmentation des familles monoparentales, dans la mesure où la séparation et le divorce peuvent intervenir avant toute naissance. Ceci étant, le nombre de ces familles a considérablement augmenté durant cette période. De 480000 en 1971, elles passent à 714000 en 1981, soit une augmentation de près de $50 \%$. Cependant, cette augmentation est relative si on la rapporte à celle de l'ensemble des familles, soit $25 \%$ pendant la même période. Autre indicateur, le phénomène concerne très majoritairement des femmes. Sont ensuite présentées de nombreuses données au sujet de la répartition de ces familles selon les situations familiales, le nombre d'enfants à charge, les niveaux de ressources, etc.

A. M. Ambert présente ensuite une revue critique et les résultats d'une recherche longitudinale concernant l'adaptation des parents gardiens et de leurs enfants. Après avoir constaté que la littérature s'intéresse traditionnellement aux effets de l'absence d'un des parents sur les enfants, elle montre que le facteur déterminant en ce domaine est moins la séparation ou le sexe du parent gardien que le niveau socio-économique et culturel. Ce qui apparaît ici comme un truisme est pourtant loin d'être pris en compte dans les recherches sur le sujet.

B. Schlesinger propose, quant à lui, une revue de littérature sur le thème des jeunes mères célibataires au Canada. Après avoir décrit l'ampleur du phénomène dans les années 80 , l'auteur présente les résultats de travaux concernant à la fois les tentatives de réduire les problèmes de grossesse chez ces jeunes femmes (pratiques contraceptives et d'interruption volontaire de grossesse), les rapports maintenus ou non avec les pères supposés des enfants, les soutiens fournis par le réseau familial, les attitudes vis-à-vis de l'adoption des enfants mis au monde par ces jeunes mères, etc. II propose ensuite de tracer quelques pistes de recherche encore peu investiguées comme : le père de l'enfant et sa famille, 
la vie quotidienne de ces jeunes mères et son suivi dans le temps, les tensions provoquées par ces situations, l'abandon des enfants par ces mères durant les premières années de vie, les effets économiques et sociaux des interventions et soutiens de ces mères, etc.

S. M. H. Hanson aborde ensuite un problème fort peu étudié, mais qui tend à se développer, à savoir les pères gardant seuls leur(s) enfant(s). L'évolution des mentalités et des pratiques sociales en ce domaine est en effet considérable. Après avoir noté qu'historiquement la garde des enfants était plutôt traditionnellement confiée aux mères dans le passé, l'auteur souligne les divers facteurs qui contribuent à renouveler les rôles et les stéréotypes sexuels. Même si aujourd'hui encore la garde en cas de séparation ou de divorce est majoritairement confiée à la mère, un mouvement s'amorce pour renouveler ce phénomène. Ceci est lié à la fois à l'entrée des femmes sur le marché du travail, assumant donc leur responsabilité de pourvoyeur pour le foyer, et à l'évolution des mentalités concernant le rôle de paternage des enfants. Les gardes partagées ou conjointes, qui tendent à se multiplier, participent du même processus. L'auteur présente alors l'ampleur quantitative de ce phénomène et un ensemble de travaux récents qui décrivent les caractéristiques de ces pères gardiens (en termes de statut socio-économique, d'âge, de race, de religion, d'emploi, etc.) et de leurs enfants. Sont également abordés les problèmes auxquels ces parents sont confrontés dans la vie quotidienne, dans la mesure où ils doivent assumer le fameux double rôle et la non moins fameuse double journée. L'auteur évoque enfin les répercussions de ces pratiques au niveau des politiques sociales et des programmes d'intervention.

G. Nelson présente dans un dernier chapitre une revue de littérature critique au sujet de l'adaptation de la famille (adultes et enfants) suite à la séparation ou au divorce. L'auteur nous montre ainsi l'ambiguïté des nombreux travaux et résultats obtenus sur ce sujet. La plupart d'entre eux font en effet l'inventaire des dégâts produits par la séparation et le divorce en négligeant cependant de tenir compte, d'une part, des variables socio-économiques pourtant fort importantes comme le rappelait A.M. Ambert, et, de l'autre, des évolutions dans le temps. Pendant de très nombreuses années, les approches longitudinales ont été radicalement négligées. On commence seulement à percevoir, semble$t-i l$, que ces situations sont susceptibles de présenter des dimensions développementales pour les sujets impliqués, mais aussi à nuancer les conclusions à la lumière des évolutions sur de longues périodes. Dans ce chapitre, l'auteur nous donne accès aux très nombreux problèmes méthodologiques en présence et nous invite à beaucoup de précautions dans nos conclusions. Les effets de la séparation et du divorce sur les personnes représentent une préoccupation politique et sociale d'importance, qui risque cependant de nous aveugler par la nécessité ou la volonté de régler des problèmes, alors même qu'ils ne se posent pas de la même manière pour tous ces foyers.

La masse documentaire recensée et la rigueur des commentaires et synthèses proposés font de ce document une véritable mine d'informations. Mieux, à la lumière de ce recensement, on peut pratiquement parler d'une histoire de la pensée scientifique sur le sujet. Ce faisant, ces auteurs nous invitent à beaucoup 
de modestie et nous rappellent, si besoin est, qu'en ce domaine, il est bien difficile de penser autre chose que ce qui est pensable à un moment donné. Audelà des pratiques sociales, la famille reste un objet fortement idéologisé et un objet politique.

Centre de recherche sur le travail social,

Claude MARTIN

Université de Caen, France. 